\title{
Prediction of pandemic risk for animal-origin coronavirus using a deep learning method
}

\author{
Zheng Kou ${ }^{1 *}\left(\mathbb{D}\right.$, Yi-Fan Huang ${ }^{1}$, Ao Shen ${ }^{1}$, Saeed Kosari ${ }^{1}$, Xiang-Rong Liu²* and Xiao-Li Qiang ${ }^{1}$
}

\begin{abstract}
Background: Coronaviruses can be isolated from bats, civets, pangolins, birds and other wild animals. As an animalorigin pathogen, coronavirus can cross species barrier and cause pandemic in humans. In this study, a deep learning model for early prediction of pandemic risk was proposed based on the sequences of viral genomes.

Methods: A total of 3257 genomes were downloaded from the Coronavirus Genome Resource Library. We present a deep learning model of cross-species coronavirus infection that combines a bidirectional gated recurrent unit network with a one-dimensional convolution. The genome sequence of animal-origin coronavirus was directly input to extract features and predict pandemic risk. The best performances were explored with the use of pre-trained DNA vector and attention mechanism. The area under the receiver operating characteristic curve (AUROC) and the area under precision-recall curve (AUPR) were used to evaluate the predictive models.
\end{abstract}

Results: The six specific models achieved good performances for the corresponding virus groups ( 1 for AUROC and 1 for AUPR). The general model with pre-training vector and attention mechanism provided excellent predictions for all virus groups (1 for AUROC and 1 for AUPR) while those without pre-training vector or attention mechanism had obviously reduction of performance (about 5-25\%). Re-training experiments showed that the general model has good capabilities of transfer learning (average for six groups: 0.968 for AUROC and 0.942 for AUPR) and should give reasonable prediction for potential pathogen of next pandemic. The artificial negative data with the replacement of the coding region of the spike protein were also predicted correctly (100\% accuracy). With the application of the Python programming language, an easy-to-use tool was created to implements our predictor.

Conclusions: Robust deep learning model with pre-training vector and attention mechanism mastered the features from the whole genomes of animal-origin coronaviruses and could predict the risk of cross-species infection for early warning of next pandemic.

Keywords: Coronavirus, Pandemic risk, Viral genome, Deep learning

\footnotetext{
*Correspondence: kouzhengcn@foxmail.com; xrliu@xmu.edu.cn

${ }^{1}$ Institute of Computing Science and Technology, Guangzhou University,

Guangzhou 510006, China

2 Department of Computer Science, Xiamen University, Xiamen 361005 , China
}

\begin{abstract}
Background
Coronaviruses $(\mathrm{CoV})$ are a group of RNA viruses whose linear, positive-sense, single-stranded RNA genomes are the longest among the known RNA viruses. The International Committee on Taxonomy of Viruses (ICTV) classifies coronaviruses into $\alpha, \beta, \gamma$, and $\delta$ genera [1]. Seven types of coronaviruses that infect humans have been identified: human coronavirus $(\mathrm{HCoV})$ 229E, OC43, NL63, and HKU1; severe acute respiratory syndrome coronavirus (SARS-CoV and SARS-CoV-2); and Middle East respiratory syndrome coronavirus (MERS-CoV).
\end{abstract}

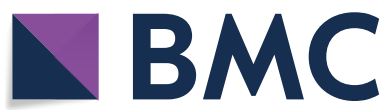

(c) The Author(s) 2021. Open Access This article is licensed under a Creative Commons Attribution 4.0 International License, which permits use, sharing, adaptation, distribution and reproduction in any medium or format, as long as you give appropriate credit to the original author(s) and the source, provide a link to the Creative Commons licence, and indicate if changes were made. The images or other third party material in this article are included in the article's Creative Commons licence, unless indicated otherwise in a credit line to the material. If material is not included in the article's Creative Commons licence and your intended use is not permitted by statutory regulation or exceeds the permitted use, you will need to obtain permission directly from the copyright holder. To view a copy of this licence, visit http://creativecommons.org/licenses/by/4.0/. The Creative Commons Public Domain Dedication waiver (http://creativeco mmons.org/publicdomain/zero/1.0/) applies to the data made available in this article, unless otherwise stated in a credit line to the data. 
SARS-CoV, MERS-CoV, and SARS-CoV-2 are highly contagious and have caused three epidemics or pandemics this century $[2,3]$.

The coronaviruses responsible for pandemics are animal-origin pathogens transmitted to humans through the intermediate host [4-7]. The intermediate hosts of SARS-CoV and MERS-CoV were civets and dromedaries, respectively $[8,9]$. The direct host of SARS-Cov-2 is not clear but is closely related to bats and pangolins [10, 11]. Coronaviruses are capable of cross-species infection through accumulation of point mutation and recombination of their RNA genome [2]. All coronaviruses responsible for epidemics or pandemics come from wild animals, are spread through respiratory droplets and close contact, and can cause severe pneumonia. Every outbreak of the coronavirus with the novelty of viral antigens has caused severe economic and societal damage. Consequently, we urgently need to develop a prediction model of the pandemic risk for human coronavirus infection and improve the prevention and control of infectious diseases for next pandemics.

The spike protein on the surface of virus particle is the most important surface membrane protein of coronaviruses, being responsible for their binding to the host cell membrane receptor and membrane fusion. It plays a very important role in cross-species infection [12]. The adaptation of other viral proteins to the internal environment of new host also affects viral replication [13]. These facts need to be considered when modeling viral infection, and artificial genome data should be used to increase the weight of the spike protein and build a robust model.

Deep learning developed rapidly in recent years, which has triggered changes in application fields such as speech recognition, image understanding, natural language processing (NLP). A recurrent neural network (RNN) is a neural network used to process sequence data and has the ability to capture the inherent characteristics of time series [14]. The original RNN model is affected by problems of gradient disappearance or explosion, proposing the long short-term memory (LSTM) network and gated recurrent unit (GRU) network, respectively $[15,16]$. At present, RNN and its variants have achieved great success in speech recognition and text translation [17]. Because genomes are also long chains comprising four alphabet units, RNNs can learn and extract the features of biological sequences. A bidirectional GRU was constructed to predict the binding preference of RNA and protein [18]. An RNN combined with an attention mechanism to predict enhancer-promoter interactions in human genes and achieved good performance [19].

The pandemic risk for animal-origin coronavirus is closely related to variations within the viral genome. We used the natural language model to construct a prediction model based on the phenotype of infection, named Coronavirus Cross-species Infection with Deep Learning (CCSI-DL), which models and analyzes six types of coronaviruses. It uses a one-dimensional (1D) convolution to extract the local features of the sequences, a GRU network to extract the long-term dependence of the sequence in two directions, and an enhanced attention mechanism to capture the weight of key features [19]. The model predicts the cross-species infection risk of animal-origin coronaviruses with well performance and can be used for early warning of pandemic risk.

\section{Methods}

\section{Initial virus data}

Coronavirus sequences were accessed from the Coronavirus Genome Resource Library (https://ngdc.cncb.ac.cn/ ncov/) on June 30, 2020, including those of MERS-CoV, HCoV-OC43, HCoV-NL63, HCoV-229E, HCoV-HKU1, SARS-CoV (combined SARS-CoV-1 and SARS-CoV-2) genome sequences and animal-origin coronaviruses [20]. A total of 3257 genomes were downloaded and humanand animal-origin coronaviruses were regarded as positive and negative samples, respectively (see Additional file 1). Using the $K$-nearest neighbors algorithm $(k=5)$, the negative samples (animal-origin coronaviruses) were divided according to the six types of positive samples (Quad-nucleotide frequency as features; Six human groups: MERS-CoV, HCoV-OC43, HCoV-NL63, HCoV229E, HCoV-HKU1, SARS-CoV), then combined with the corresponding positive samples to form six types of coronavirus data sets (see Additional file 2).

\section{Artificial negative data}

The spike protein is the most important surface membrane protein of coronaviruses and is responsible for their binding to the receptor of host cell, which plays the key role in transmission efficiency and host range [13]. If the spike protein of positive virus is replaced with that of negative virus (see Additional file 3), the positive virus should significantly decrease transmission efficiency and its phenotype label should be changed [13]. According to the strategy of artificial recombination in silicon, the generated sequences based on the replacement of the coding region of the spike protein for the initial positive sample were added to the negative sample data set (see Additional file 4). During training, the weights of the spike protein were further increased in the model, thereby improving the prediction accuracy with robustness. Considering the synergistic effect of other viral proteins on cross-species infection, this approach is consistent with biological studies of host adaption [13]. After the addition of artificial negative data and the balance of 
sample number (direct duplication), the final dataset of six viruses was shown in Table 1.

\section{Framework of the CCSI-DL model}

The CCSI-DL model consists of five main steps: genome segmentation, sequence embedding, 1D convolution, the RNN, and the attention mechanism. Figure 1 shows the structure of the proposed model in the paper.

\section{Sequence segmentation and embedding}

Genome sequence of coronavirus cannot be directly used for model construction. The method of traditional sequence conversion uses one-hot vectors to encode DNA sequence fragments. Because the information between each vector is independent, the model cannot capture the hidden associated information in the sequence and is therefore unsuitable for deep learning algorithms. To avoid this problem, we used the DNA vector obtained by the dna2vec method [21] as the pretraining vector. The dna2vec method is based on the word2vec word embedding model [22], which is the classical text representation method in the NLP field.

The dna2vec method uses DNA sequence fragments of length $k$ ( $k$-mers) as words. Its purpose is to calculate the distributed representation of DNA fragments and capture the associated information in the original sequence. Based on the pre-training vector, the model uses fine-tuning strategies in the embedding layer to improve the performance of the model [23]. In the paper, we chose $k=2$ and an embedding dimension of 8 [19].

The length of the original genome sequence of the coronavirus is about $27-32 \mathrm{~kb}$. We took a two-base RNA fragment as a basic word and preprocessed the original genome sequence to obtain a numerical index sequence. To facilitate model input, the obtained index sequence was rounded to a length of $30 \mathrm{~kb}$ and divided into 10 equal segments ( $3 \mathrm{k}$ for each segment). The embedding layer of the model performed embedding of the input sequence based on the pre-trained vector. To improve the performance of the model, the weight of the embedding layer was set to be trainable so that the DNA vector could be fine-tuned according to the training data of coronavirus genome.

\section{One-dimensional convolution}

The CCSI-DL model combines a 1D convolution with an RNN for feature extraction. A 1D convolution is firstly used to capture the local correlation features in the sequence and then input into the bidirectional GRU network to extract the global correlation features. The
Table 1 Genomic data for six coronaviruses

\begin{tabular}{lcc}
\hline & Positive samples, $\boldsymbol{n}$ & $\begin{array}{l}\text { Negative } \\
\text { samples, } \boldsymbol{n}\end{array}$ \\
\hline MERS-CoV & 1044 & 1138 \\
HCoV-OC43 & 482 & 534 \\
HCoV-NL63 & 240 & 262 \\
HCoV-229E & 240 & 262 \\
HCoV-HKU1 & 390 & 387 \\
SARS-CoV & 638 & 587 \\
\hline
\end{tabular}

1D convolution slides along the data in one dimension and extracts features from shorter segments in the genome sequence.

For computer vision tasks, using a deeper 2D convolution model can produce a more accurate classification [24], but increasing the network depth does not necessarily improve performance for $1 \mathrm{D}$ data [25]. The 1D convolutional layer is used to extract the local features of the sequence after embedding. We set the number of convolution kernels (filters) to 4 and the length of convolution kernels (kernel_size) to 50, and used the rectified linear activation function (ReLU). The ReLU can give the sparsity for the network and the pooling layer can reduce the computational complexity. The maximum pooling layer was connected after the convolutional layer to further reduce the sequence length, increase the calculation speed, and avoid overfitting.

\section{Recurrent neural network}

The recurrent unit used in the RNN was the GRU network, which is improved by the LSTM network and can also solve problems such as long-term memory and gradients in back propagation [26]. The GRU removes the cell state from the LSTM network and retains two gated units (the update gate and the rest gate), which simplifies the LSTM structure and reduces training complexity while achieving a similar experimental effect.

To capture characteristics of biology sequences adequately, a bidirectional GRU architecture was used (two unidirectional GRU layers, one forward and one backward). This architecture connects the forward hidden state and the reverse hidden state, enabling the output result to simultaneously account for the sequence correlation of the forward and backward states. The output of a single GRU layer was set to 50 dimensions, and the two opposing outputs were connected to obtain a total output dimension of 100 . 


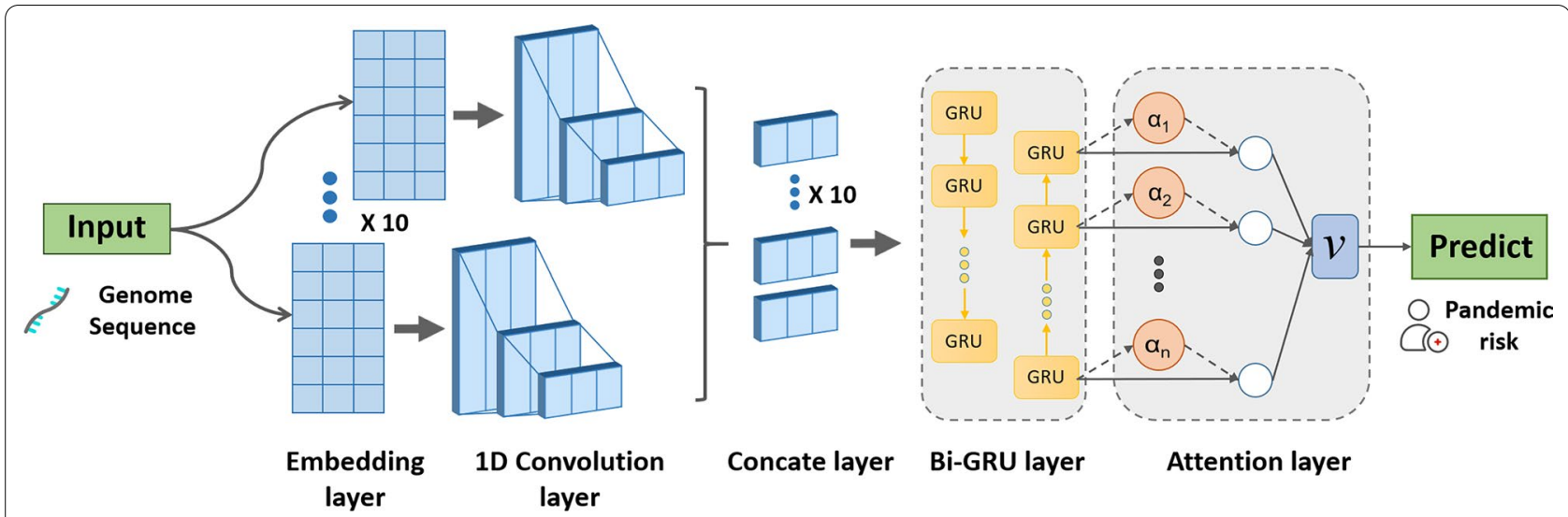

Fig. 1 Flowchart of the deep learning method. The Coronavirus Cross-species Infection with Deep Learning (CCSI-DL) model consists of five main steps: genome segmentation, sequence embedding, one-dimensional convolution, the recurrent neural network, and the attention mechanism

\section{Attention mechanism}

Following the bidirectional GRU layer, the attention layer is used to learn the weights of features. Inspired by human attention, its purpose is to focus attention on a specific part based on a large amount of information. The attention mechanism was first applied in the field of image processing, then introduced into the NLP field for machine translation in 2015 [27] and extended to various other NLP tasks, resulting in many improvements [28]. Assuming that the features in the sequence are not equally important to the prediction, using the attention mechanism can improve the contribution of key features to the prediction. The attention mechanism is described by the following formulae [19]:

$$
\begin{aligned}
& h_{i}=\tanh \left(\mathrm{W}_{\omega} \mathrm{f}_{\mathrm{i}}+\mathrm{b}_{\omega}\right) \\
& \alpha_{i}=\frac{\exp \left(\mathrm{h}_{\mathrm{i}}^{\mathrm{T}} \mathrm{h}_{\omega}\right)}{\sum_{i} \exp \left(\mathrm{h}_{\mathrm{i}}^{\mathrm{T}} \mathrm{h}_{\omega}\right)} \\
& v=\sum_{i} \alpha_{i} f_{i}
\end{aligned}
$$

For the $i$ th feature $f_{i}$ output by the previous layer, its hidden representation $h_{i}$ is calculated. The importance of features is measured by the similarity between $h_{i}$ and the context vector $h_{w}$. The normalized weight $\alpha_{i}$ of each feature is obtained by multiplying $h_{w}$ by $h_{i}$ and then using the softmax function. The feature vector $f_{i}$ is multiplied by the corresponding weight $\alpha_{i}$ and summed to the final output vector $v$ used for prediction. $W_{\omega}, b_{\omega}$, and $h_{\omega}$ were randomly initialized and learned during model training.

\section{Model training and evaluation}

During training, we used a batch size of 24, the crossentropy loss function, and the Adam optimization algorithm to update the network weights. To avoid overfitting, a batch normalization layer and a dropout layer (random dropout probability $=0.5$ ) were added after the merge layer. After amplification of positive samples, the six virus data sets were randomly divided into a training set $(90 \%)$ and a test set $(10 \%)$. The model was trained using the training set for 15 rounds, then the effect of the model training was evaluated using the test set.

To evaluate the predictive models, we calculated the area under the receiver operating characteristic curve (AUROC) [29] and the area under precision-recall curve (AUPR) [30], both of which are suitable for imbalanced data sets. The receiver operating characteristic (ROC) curve plots the true positive rate as a function of the false positive rate. The closer the AUROC is to 1 , the better the performance of the model. The ROC curve is not affected by the distribution of positive and negative samples, so AUROC is suitable as an evaluation index for an unbalanced binary classification model. The precisionrecall curve plots the precision as a function of the recall, reflecting the trade-off between the model's accuracy in identifying positive examples. The closer the AUPR value is to 1 , the better the performance of the model.

To verify the advantage of pre-trained DNA vector and attention mechanism on the performance of CCSI-DLspecific and CCSI-DL-general, three variant models were proposed: (1) CCSI-DL-nopre, in which the embedding layer does not use pre-training DNA vectors; (2) CCSIDL-onehot, which does not use an embedding layer and only uses a one-hot-coded sequence as input; (3) CCSIDL-noatt, which does not use the attention layer. The same training processes were applied to specific and 
general models: CCSI-DL-spe-nopre, CCSI-DL-spe-onehot, CCSI-DL-spe-noatt, CCSI-DL-gen-nopre, CCSI-DLgen-onehot, and CCSI-DL-gen-noatt.

\section{Implementation of the prediction tool}

We used the Python 3.7.4, tensorflow 2.1.0 and Keras 2.3.1 to create an easy-to-use tool that implements our predictor, which is freely accessible via https://github. com/kouzheng/CCSI-DL and can run in an end-to-end way and handle massive data. Users need to prepare the query sequences of coronavirus genome in the FASTA format, input the name for query file, and set confidence parameter (from 0.0 to 1.0 ) before running the tool. Setting a smaller confidence parameter results in more sensitive predictions. A predicted phenotype of pandemic risk is labeled " $\mathrm{H}$ ", while the label " $\mathrm{N}$ " indicates no transmission.

\section{Results}

Specific model for each virus group

As there are genetic differences in the sequences of the six virus types, we created separate prediction models using the genome data set for each of the six viruses (called CCSI-DL-specific) and evaluated the generalizability of each specific model to other virus groups. The AUROC and AUPR values obtained after training the six specific models were shown in Table 2 . The best results ( 1 for AUROC and 1 for AUPR) were obtained when the model was trained and tested using the same group of virus data set, while the predictive performances using test data sets from different virus groups was reduced significantly. The MERS-CoV model achieved well performance for the MERS-CoV data ( 1 for AUROC and 1 for AUPR) and bad prediction for the HCoV-HKU1 data (0.03 for AUROC and 0.333 for AUPR), the HCoV-NL63 data ( 0.335 for AUROC and 0.375 for AUPR). The SARS$\mathrm{CoV}$ model had excellent performance for the SARS-CoV and MERS-CoV data and low prediction for the $\mathrm{HCoV}$ OC43 data (0.946 for AUROC and 0.887 for AUPR),
HCoV-NL63 data (0.96 for AUROC and 0.881 for AUPR) and $\mathrm{HCoV}-229 \mathrm{E}$ data $(0.863$ for AUROC and 0.734 for AUPR).

\section{General model for all virus groups}

Training specific models for six coronavirus data sets is time-consuming and has poor generalizability, hence we need to build a general model for all virus groups. We trained a general model, called CCSI-DL-general, by combining the training data of six virus groups and was then evaluated for each virus group. The model was trained using the mix training data set for 15 rounds, then the performance of the general model was evaluated using the test set. As a result, the CCSI-DL-general model provided very good predictions for all of virus groups with the artificial negative data ( 1 for AUROC and 1 for AUPR).

\section{Advantage of pre-training vector and attention mechanism}

As shown in Fig. 2A, B, the performances of three variant models of CCSI-DL-specific were not as good as those of the original model. Without the use of pre-trained DNA vector, the values of AUROC and AUPR for $\mathrm{HCoV}$ 229E and HCoV-HKU1 were obviously decreased (about $10-20 \%$ reduction). With the use of one-hot coding, the specific model for MERS-CoV and SARS-CoV achieved low performance. Without the use of attention mechanism, the performance for the MERS-CoV model slightly reduced while that of the $\mathrm{HCoV}-229 \mathrm{E}$ reduced obviously ( 0.91 for AUROC and 0.837 for AUPR). According to the results shown in Fig. 2A, B, it was suggested that the six virus models get the best performance with the use of pre-trained DNA vector and attention mechanism.

The performance of three variant models of general model was shown in Fig. 2C, D. The CCSI-DL-gennopre model had slight reduction of performance for all of the six viruses. The CCSI-DL-gen-onehot model had obviously reduction of performance for MERS-CoV,

Table 2 Prediction performance of CCSI-DL-specific model for pandemic risk

\begin{tabular}{|c|c|c|c|c|c|c|c|c|c|c|c|c|}
\hline \multirow[t]{2}{*}{ Train \Test } & \multicolumn{2}{|c|}{ MERS-CoV } & \multicolumn{2}{|c|}{ HCoV-OC43 } & \multicolumn{2}{|c|}{ HCoV-NL63 } & \multicolumn{2}{|c|}{ HCoV-229E } & \multicolumn{2}{|c|}{ HCoV-HKU1 } & \multicolumn{2}{|c|}{ SARS-CoV } \\
\hline & AUROC & AUPR & AUROC & AUPR & AUROC & AUPR & AUROC & AUPR & AUROC & AUPR & AUROC & AUPR \\
\hline MERS-CoV & 1 & 1 & 0.566 & 0.474 & 0.335 & 0.375 & 0.503 & 0.441 & 0.03 & 0.333 & 0.984 & 0.942 \\
\hline HCoV-OC43 & 0.066 & 0.328 & 1 & 1 & 1 & 1 & 0.528 & 0.454 & 0.943 & 0.896 & 0.331 & 0.396 \\
\hline HCoV-NL63 & 0.262 & 0.375 & 1 & 1 & 1 & 1 & 0.644 & 0.523 & 0.895 & 0.815 & 0.549 & 0.488 \\
\hline HCoV-229E & 0.516 & 0.471 & 0.917 & 0.808 & 0.995 & 0.995 & 1 & 1 & 0.173 & 0.366 & 0.79 & 0.645 \\
\hline HCoV-HKU1 & 0.432 & 0.436 & 0.914 & 0.924 & 1 & 1 & 0.66 & 0.534 & 1 & 1 & 0.621 & 0.536 \\
\hline SARS-COV & 1 & 1 & 0.946 & 0.887 & 0.96 & 0.881 & 0.863 & 0.734 & 1 & 1 & 1 & 1 \\
\hline
\end{tabular}


$\mathrm{HCoV}-\mathrm{OC} 43, \mathrm{SARS}-\mathrm{CoV}$ and $\mathrm{HCoV}-229 \mathrm{E}$. The low performance for $\mathrm{HCoV}-229 \mathrm{E}$ is obvious $(0.996$ for AUROC and 0.967 for AUPR). Without the use of attention mechanism, all of the virus groups achieved slightly performance reduction except $\mathrm{HCoV}-229 \mathrm{E}$ (0.911 for AUROC and 0.875 for AUPR). The CCSI-DL-gen-noatt model had poor prediction for $\mathrm{HCoV}-229 \mathrm{E}$, which only had 0.911 for AUROC and 0.875 for AUPR. According to the results shown in Fig. 2C, D, it was suggested that the CCSI-DLgeneral model with the use of pre-trained DNA vector and attention mechanism get the best performance.

\section{Advantage of artificial negative data}

Compared with the genome sequences of original positive data, those of artificial negative data remain unchanged except the coding region of the spike protein. The data constrain for the spike protein is compatible with animal tests, which will increase the weight of the spike protein and will benefit for robust prediction. To show the advantage of data constrain based on biology trials, we used the original virus data to repeat the training of CCSI-DL-specific and CCSI-DL-general and predict the artificial negative data.

Table 3 shows the predictions of CCSI-DL-specific and CCSI-DL-general for the artificial negative data. It can be seen that the model trained with the initial data of coronavirus genome cannot correctly predict the artificial negative data and give positive output with thoroughly errors (about 0 for accuracy). Because the infection efficiency for the "positive" virus is vastly reduced when the encoding region of the spike protein is replaced by that of the "negative" virus, adding artificial data to the original data set for training will improve the weight of the spike protein and increase the robustness of the model.

\section{Transfer learning ability of general model}

The CCSI-DL-general model achieved excellent performance as shown above. To determine whether CCSI-DLgeneral can achieve good performance using a new data set, we fine-tuned the parameters and assessed its transfer learning ability by using a certain virus as the new data set (Dnewtrain and Dnewtest) and mixed the genome data of the other five viruses as a combined training set (Dtrain). The CCSI-DL-general model was pre-trained for 15 rounds using Dtrain, trained for 10 rounds using Dnewtrain, and evaluated using Dnewtest. The model for the evaluation of transfer leering ability was named after CCSI-DL-transfer.

As shown in Fig. 3, the performance of CCSI-DLtransfer was compared with that of CCSI-DL-general. The CCSI-DL-transfer model achieved excellent performance for $\mathrm{HCoV}-\mathrm{OC} 43, \mathrm{HCoV}-\mathrm{NL} 63, \mathrm{HCoV}-\mathrm{HKU} 1$ and SARS-CoV (almost 1 both for AUROC and AUPR). The

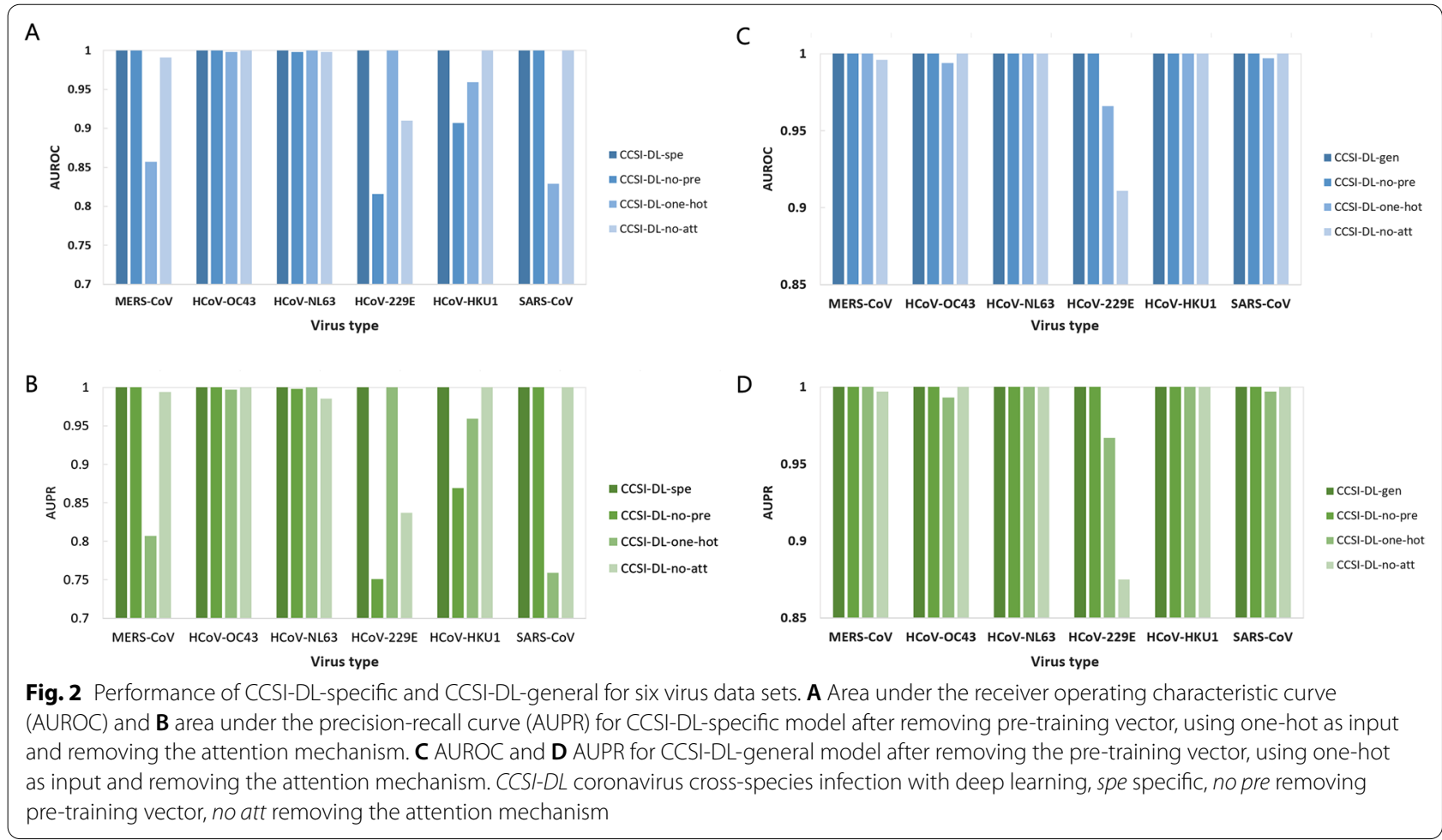


MERS-CoV had slight reduction of prediction performance (0.993 for AUROC and 0.996 for AUPR). However, the low performance for HCoV-229E was got with 0.818 for AUROC and 0.657 for AUPR. The main reason for the performance of HCoV-229E is about small number of virus data and high identity of genome sequence, which should be conquered with the increase of genome sequences in public database. The excellent performance for most coronavirus (average score for six groups: 0.968 for AUROC and 0.942 for AUPR) proved that the CCSIDL-general model can be used as a pre-training model for transfer learning and will give the reasonable prediction for next pandemic pathogen.

\section{Discussion}

Coronavirus is an animal-origin pathogen that can cause disease in humans [31, 32]. A model for phenotype identification of notorious disease is urgently needed to be developed [33, 34]. In the paper, we present a deep learning model of cross-species coronavirus infection that combines a one-dimensional convolutional neural network with a bidirectional gated recurrent unit network. This deep learning method strengths the weight of the spike protein and provides an effective model for early warning of cross-species infections.

Although the spike protein is responsible for their binding to the host cell membrane receptor and play the key role in the cross-species transmission, the synergistic effect of other viral proteins reported by biological studies of host adaption should be considered [13]. The one-dimensional convolution was used to extract the sequence features of genome sequences and the recurrent neural network with the attention mechanism was used to build the predictive model. Moreover, artificial negative data with genome recombination in the coding region of spike protein improve its weight and increase the robustness of the model.

The results about six specific models show that they are able to learn the sequence features in a single virus data set and cannot provide good predictive performances for other virus groups. However, it should be noted that the

Table 3 Performance of CCSI-DL-specific and CCSI-DL-general against artificial negative data

\begin{tabular}{llllcc}
\hline Model $^{\text {a }}$ & MERS-CoV & HCoV-OC43 & HCoV-NL63 & HCoV-229E & HCoV-HKU1 \\
\hline Specific & 0.004 & 0 & 0 & 0.033 & 0 \\
General & 0 & 0 & 0 & 0 & 0 \\
\hline
\end{tabular}

${ }^{\text {a }}$ Test data were wholly negative sample and the accuracy was selected as an evaluation metric. CCSI-DL coronavirus cross-species infection with deep learning

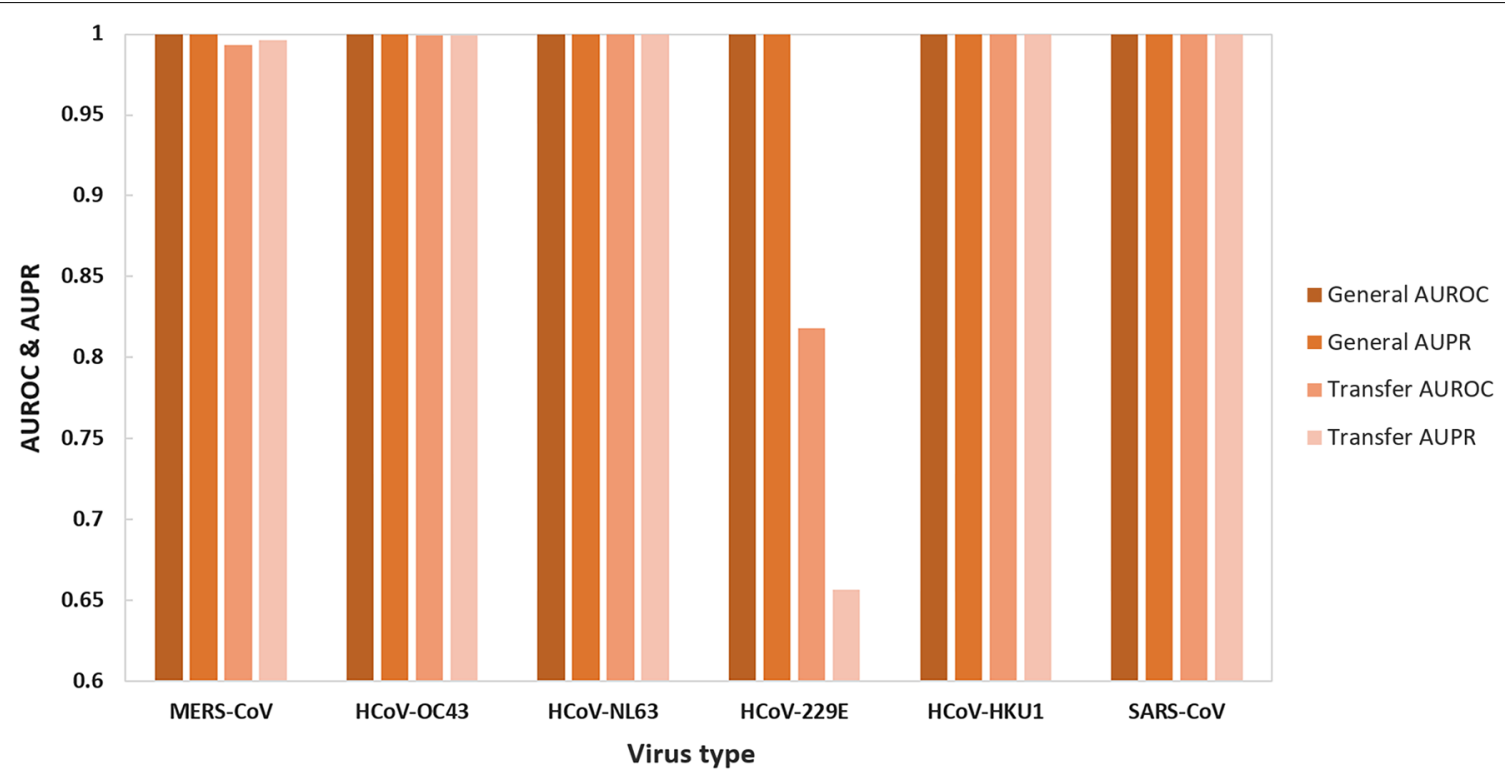

Fig. 3 Performance of CCSI-DL-general model. Performance of the pre-trained CCSI-DL-general model transferred to a new data set. AUROC area under the receiver operating characteristic curve, AUPR area under precision-recall curve, CCSI-DL coronavirus cross-species infection with deep learning 
SARS-CoV model and MERS-CoV model provided well performance for each other. The similar result is suitable for the HCoV-OC43 model and the HCoV-NL63 model. The potential reason is that the similar mechanism for cross-species infection was employed.

The proposed deep learning method used the pretrained DNA vector and attention mechanism to extract features of coronavirus genomes. Based on the evaluation of transfer learning and artificial negative data, it was proved that the general model is robust and reasonable. We used the Python programming language to create a powerful tool to benefit the surveillance for public health. Moreover, we try this tool to predict SARS-Cov-2 genome data from Brazil, United Kingdom, South Africa, and India (Positive sample; Mutant human-origin virus) and achieved $100 \%$ predictive accuracy.

The length of the genome sequence of the coronavirus is about $27-32 \mathrm{~kb}$. In the paper, the long sequences of viral genomes were totally considered and divided into ten segments to increase the performance of the prediction model. However, the main limitation of the proposed method in the paper is that the interpretability of predictive results was reduced because the convolutional method was used to extract features of viral genomes and the relation between sequence features and prediction results was not obvious. The attention matrix should be used to analysis the correlation of ten genome segments. The weights of convolution network should be emphasized to master the key region in the genome sequences. Although the end-to-end model was easy to extract the feature and flexible to build the model, the development about interpretability of prediction output should be considered in the future [35], which will increase the understanding of the mechanism about cross-species coronavirus infection.

\section{Conclusions}

We proposed a CCSI-DL model, which combines a bidirectional GRU with a one-dimensional convolution and uses the genome sequence of coronaviruses as direct input to predict the pandemic risk of human infection. We trained and tested the CSSI-DL model using singleand multi-group coronavirus genome data and achieved good performances ( 1 for AUROC and 1 for AUPR). Retraining experiments showed that the model has good transfer learning capabilities and the artificial negative data with genome recombination in the coding region of spike protein were correctly predicted. In contrast to traditional machine learning methods, deep learning models master the features from the whole genome of coronavirus and predict the risk of cross-species viral infections with robustness.

\section{Abbreviations}

CoV: Coronavirus; ICTV: International Committee on Taxonomy of Viruses; HCoV: Human coronavirus; SARS-CoV: Severe acute respiratory syndrome coronavirus; MERS-CoV: Middle East respiratory syndrome coronavirus; NLP: Natural language processing; RNN: Recurrent neural network; LSTM: Long short-term memory; GRU: Gated recurrent unit; 1D: One-dimensional; ReLU: Rectified linear activation function; CCSI-DL: Coronavirus cross-species infection with deep learning; ROC: Receiver operating characteristic curve; AUROC: Area under the receiver operating characteristic curve; AUPR: Area under precision-recall curve.

\section{Supplementary Information}

The online version contains supplementary material available at https://doi. org/10.1186/s40249-021-00912-6.

Additional file 1. The number for the initial virus data.

Additional file 2. The dataset for six virus types.

Additional file 3. The Entry ID for the selected negative virus to recombine initial positive virus.

Additional file 4. The number of virus data with artificial negative data.

\section{Acknowledgements}

We would like to acknowledge the originating and submitting laboratories of the viral sequences from the Coronavirus Genome Resource Library.

\section{Authors' contributions}

$Z K, X L$ and $X Q$ designed the framework of analysis. $Y H$ and SK performed all computational work. YH and AS implemented the code and software. ZK and $\mathrm{YH}$ wrote the manuscript. All authors read and approved the final manuscript.

\section{Funding}

This work was supported by the National Natural Science Foundation of China $(61972109,62172114,61632002)$.

\section{Availability of data and materials}

A total of 3257 coronavirus genomes analyzed during the current study are available in the Coronavirus Genome Resource Library (https://ngdc.cncb.ac. $\mathrm{cn} / \mathrm{ncov} /$ ) [20]. The number for the initial virus data is provided as Additional file 1. The nomenclature for six virus types is provided as Additional file 2. The Entry ID for the selected negative virus to recombine initial positive virus is provided as Additional file 3 . The number of virus data with artificial negative data is provided as Additional file 4.

\section{Declarations}

Ethics approval and consent to participate

Not applicable.

\section{Consent for publication \\ Not applicable.}

\section{Competing interests}

The authors declare that they have no competing interests.

Received: 13 August 2021 Accepted: 11 October 2021

Published online: 24 October 2021

\section{References}

1. Gorbalenya A, Enjuanes L, Ziebuhr J, Snijder E. Nidovirales: evolving the largest RNA virus genome. Virus Res. 2006;117(1):17-37.

2. Cui J, Li F, Shi Z. Origin and evolution of pathogenic coronaviruses. Nat Rev Microbiol. 2019:17(3):181-92.

3. WHO. Coronavirus (COVID-19) Dashboard. 2021. https://covid19.who.int/, 
4. Ge X, Li J, Yang X, Chmura A, Zhu G, Epstein J, et al. Isolation and characterization of a bat SARS-like coronavirus that uses the ACE2 receptor. Nature. 2013;503:535-8

5. Hu B, Zeng L, Yang X, Ge X, Zhang W, Li B, et al. Discovery of a rich gene pool of bat SARS-related coronaviruses provides new insights into the origin of SARS coronavirus. PLoS Pathog. 2017;13:e1006698.

6. Lau S, Li K, Tsang A, Lam C, Ahmed S, Chen H, et al. Genetic characterization of Betacoronavirus lineage $C$ viruses in bats reveals marked sequence divergence in the spike protein of pipistrellus bat coronavirus HKU5 in Japanese pipistrelle: implications for the origin of the novel Middle East respiratory syndrome coronavirus. J Virol. 2013;87:8638-50.

7. Zhou P, Yang X, Wang X, Hu B, Zhang L, Zhang W, et al. A pneumonia outbreak associated with a new coronavirus of probable bat origin. Nature. 2020;579:270-3.

8. Guan Y, Zheng B, He Y, Liu X, Zhuang Z, Cheung C, et al. Isolation and characterization of viruses related to the SARS coronavirus from animals in southern China. Science. 2003;302:276-8.

9. Alagaili A, Briese T, Mishra N, Kapoor V, Sameroff S, Burbelo P, et al. Middle East respiratory syndrome coronavirus infection in dromedary camels in Saudi Arabia. MBio. 2014:5:e00884-e914

10. Lam T, Jia N, Zhang Y, Shum M, Jiang J, Zhu H, et al. Identifying SARS-CoV2-related coronaviruses in Malayan pangolins. Nature. 2020;583:282-5.

11. Xiao K, Zhai J, Feng Y, Zhou N, Zhang X, Zou J. Isolation of SARS-CoV2-related coronavirus from Malayan pangolins. Nature. 2020;583:286-9.

12. Qiang X, Xu P, Fang G, Liu W, Kou Z. Using the spike protein feature to predict infection risk and monitor the evolutionary dynamic of coronavirus. Infect Dis Poverty. 2020;9:33.

13. Menachery V, Yount B, Debbink K, Agnihothram S, Gralinski L, Plante J, et al. A sars-like cluster of circulating bat coronaviruses shows potential for human emergence. Nature Med. 2015;21:1508-13.

14. Rumelhart D, Hinton G, Williams R. Learning representations by back propagating errors. Nature. 1986;323:533-6.

15. Hochreiter S, Schmidhuber J. Long short-term memory. Neural Comput. 1997;9(8):1735-80.

16. Yao K, Cohn T, Vylomova K, Duh K, Dyer C. Depth-gated recurrent neural networks. Arxiv.org. 2015. https://arxiv.org/abs/1508.03790v1.

17. Salehinejad H, Sankar S, Barfett J, Colak E, Valaee S. Recent advances in recurrent neural networks. Arxiv.org. 2017. https://arxiv.org/abs/1801. 01078.

18. Shen Z, Deng S, Huang D. RNA-protein binding sites prediction via multi scale convolutional gated recurrent unit networks. IEEE/ACM Trans Comput Biol Bioinform. 2020;17(5):1741-50.

19. Hong Z, Zeng X, Wei L, Liu X. Identifying enhancer-promoter interactions with neural network based on pre-trained DNA vectors and attention mechanism. Bioinformatics. 2019;36(4):1037-43.
20. Zhao W, Song S, Chen M, Zou D, Ma L, Ma Y, et al. The 2019 novel coronavirus resource. Yi Chuan. 2020;42(2):212-21.

21. Patrick N. dna2vec: consistent vector representations of variable-length k-mers. Arxiv.org. 2017. https://arxiv.org/abs/1701.06279.

22. Mikolov T, Chen K, Corrado G, Dean J. Efficient estimation of word representations in vector space. Arxiv.org. 2013. https://arxiv.org/abs/1301. $3781 \mathrm{v} 1$.

23. Kim Y. Convolutional neural networks for sentence classification. Arxiv. org. 2014. https://arxiv.org/abs/1408.5882v1.

24. LeCun Y, Bengio Y, Hinton G. Deep learning. Nature. 2015:521(7553):436-44

25. Min S, Lee B, Yoon S. Deep learning in bioinformatics. Brief Bioinform. 2017;18(5):851-69.

26. Chung J, Gulcehre C, Cho K, Bengio Y. Empirical evaluation of gated recurrent neural networks on sequence modeling. Arxiv.org. 2014. https:// arxiv.org/abs/1412.3555.

27. Bahdanau D, Cho K, Bengio Y. Neural machine translation by jointly learning to align and translate. Arxiv.org. 2014. https://arxiv.org/abs/1409.0473.

28. Luong M, Pham H, Manning C. Effective approaches to attention-based neural machine translation. Proceedings of the 2015 Conference on Empirical Methods in Natural Language Processing. 2015; 1412-1421.

29. Hanley J, McNeil B. The meaning and use of the area under a receiver operating characteristic (ROC) curve. Radiology. 1982;143:29-36.

30. Davis J, Goadrich M. The relationship between precision-recall and ROC curves. Proceedings of the 23rd International Conference on Machine Learning. 2006; 233-240.

31. Fang $F$, Song $Y$, Hao L, Nie K, Sun X. A case of COVID-19 detected in a cargo worker at Pudong Airport-Shanghai Municipality, China, November 8, 2020. China CDC Weekly. 2020;2(47):910-1.

32. Zhang M, Xiao J, Deng A, Zhang Y, Zhuang Y, Hu T, et al. Transmission dynamics of an outbreak of the COVID-19 Delta variant B.1.617.2Guangdong Province, China, May-June 2021. China CDC Weekly. 2021:3(27):584-6.

33. Dhaka V, Rani G, Oza M, Sharma T, Misra A. A deep learning model for mass screening of COVID-19. Int J Imaging Syst Technol. 2021;31:483-98.

34. Rani G, Oza M, Dhaka V, Pradhan N, Verma S, Rodrigues J. Applying deep learning-based multi-modal for detection of coronavirus. Multimed Syst. 2021. https://doi.org/10.1007/s00530-021-00824-3.

35. Pan $Y$, Lei $X$, Zhang Y. Association predictions of genomics, proteinomics, transcriptomics, microbiome, metabolomics, pathomics, radiomics, drug, symptoms, environment factor, and disease networks: a comprehensive approach. Med Res Rev. 2021. https://doi.org/10.1002/med.21847.
Ready to submit your research? Choose BMC and benefit from:

- fast, convenient online submission

- thorough peer review by experienced researchers in your field

- rapid publication on acceptance

- support for research data, including large and complex data types

- gold Open Access which fosters wider collaboration and increased citations

- maximum visibility for your research: over 100M website views per year

At $\mathrm{BMC}$, research is always in progress.

Learn more biomedcentral.com/submissions 